\title{
Synthesis and Electrical Properties of $\mathrm{Bi}_{2} \mathrm{Te}_{3}$-Based Thermoelectric Materials Doped with Er, Tm, Yb, and $\mathrm{Lu}$
}

\author{
M. N. Yapryntsev*, R. A. Lyubushkin, O. N. Soklakova, and O. N. Ivanov \\ Belgorod National Research University, Belgorod, 308015 Russia \\ *e-mail:yaprintsev@bsu.edu.ru
}

Submitted December 12, 2016; accepted for publication December 19, 2016

\begin{abstract}
Nanopowders of $\mathrm{Bi}_{2} \mathrm{Te}_{3}$ and $\mathrm{R}_{0.1} \mathrm{Bi}_{1.9} \mathrm{Te}_{3}$ (where $\mathrm{R}=\mathrm{Er}$, $\mathrm{Tm}, \mathrm{Yb}, \mathrm{Lu}$ ) are obtained by microwave solvothermal synthesis. The powder-like materials are compacted by cold isostatic compression followed by annealing in argon. The influence of the doping agent on the structure and characteristics of the derived materials are investigated. It is demonstrated that the introduction of rare-earth elements $(2$ at $\%)$ into the bismuth-telluride lattice leads to a decrease in the electrical resistivity and to an increase in the Seebeck coefficient. The best thermoelectric properties are obtained for the sample of bismuth telluride doped with thulium.
\end{abstract}

DOI: $10.1134 / \mathrm{S} 106378261706029 \mathrm{X}$

\section{INTRODUCTION}

Recently, the possibility of increasing the thermoelectric efficiency of various thermoelectric materials via size effects has been extensively studied [1-4]; however, the preparation of samples containing quantum dots and quantum wells is associated with great economic and technical difficulties. Therefore, an approach based on the formation of bulk nanostructured thermoelectric materials is of great interest. In these materials, there are conditions for the emergence of various effects, a consequence of which is an increase in the thermoelectric figure of merit $Z T$. Another way to modify materials is the introduction of various doping elements into the crystal lattice. The doping of $\mathrm{Bi}_{2} \mathrm{Te}_{3}$ with lanthanides can affect the transport properties of thermoelectric materials according to following mechanisms: (i) an increase in the density of states near the Fermi level; (ii) the formation of local defects leading to the additional scattering of charge carriers; and (iii) additional scattering of charge carriers by the localized magnetic moments of rare-earth elements (REEs) [5-9].

\section{EXPERIMENTAL}

The synthesis of $\mathrm{Bi}_{2} \mathrm{Te}_{3}$ and $\mathrm{R}_{0.1} \mathrm{Bi}_{1.9} \mathrm{Te}_{3}$ powders (where $\mathrm{R}=\mathrm{Er}, \mathrm{Tm}, \mathrm{Yb}, \mathrm{Lu}$ ) was performed by the microwave solvothermal method. The proposed method of synthesis provides an opportunity for the purposeful modification of the micromorphology, properties and particle size of the synthesized materials by varying such parameters as the temperature, process duration, pressure, and solvent composition.
Microwave heating differs from traditional thermal heating by the absence of high volume and time gradients, and also by unequal influence on the components of heterogeneous systems, which differ in terms of composition.

High-purity reactants of erbium oxide, ytterbium, thulium and lutecium ("Lanhit" Company), bismuth oxide $\left(\mathrm{Bi}_{2} \mathrm{O}_{3}, 99.9 \%\right)$ and tellurium oxide $\left(\mathrm{TeO}_{2}\right.$, 99.9\%) were used in the synthesis. Ethylene glycol (1,2-ethylene glycol, 99.8\%), nitric acid $\left(\mathrm{HNO}_{3}\right.$, $70 \%), N, N$-dimethylformamide (DMF, anhydrous, $99.8 \%$ ) were also used. The synthesis of powders was carried out in a closed-type microwave reactor (MARS 6). The reaction mixture was placed in a $100-\mathrm{mL}$ reactor chamber and exposed to microwave radiation with a power of $300 \mathrm{~W}$ at $2.45 \mathrm{MHz}$. The reaction was carried out at a temperature of $180^{\circ} \mathrm{C}$ and a pressure of $4 \mathrm{MPa}$ for 20 minutes.

Phase identification in the powders and compacted samples was performed by X-ray phase analysis using a SmartLab X-ray diffractometer (RIGAKU) with $\mathrm{Cu} K_{\alpha}$-radiation and a $\mathrm{Ni}$ filter. The $2 \theta$ angle was between 10 and 80 degrees with a step of 0.01 degree and a rate of 1 degree $/ \mathrm{min}$. Phase identification was carried out using the powder X-ray diffraction database PDF (JCPDICDD), PDF-2. Lattice indexing was conducted using graphical analysis methods.

In this study, transmission electron microscopy (TEM) with the use of a Jeol 2100 transmission electron microscope (accelerating voltage of $200 \mathrm{kV}$ ) was performed to investigate the morphology, particle sizes, and their distribution in the initial powders. 


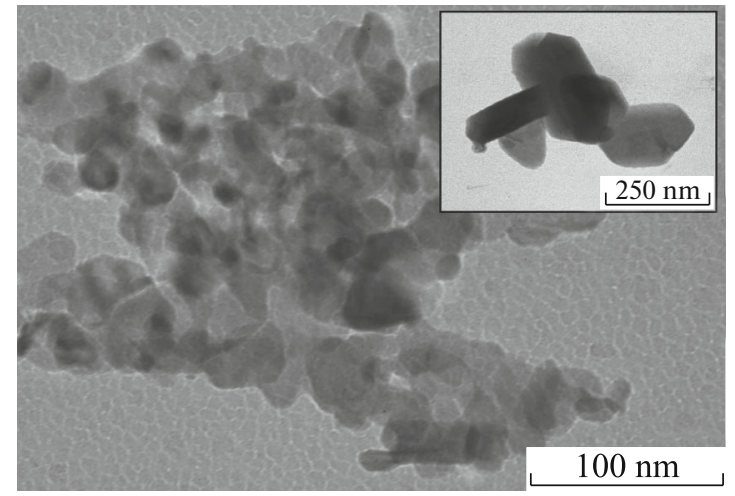

Fig. 1. Typical TEM image of the as-prepared and annealed (see the inset) powder.

Scanning electron microscopy (SEM) with the use of a Quanta 600 microscope was performed to investigate the compacted samples.

The electrical conductivity and the Seebeck coefficient were measured in the temperature range from 300 to $650 \mathrm{~K}$ using the thermoelectric-characteristic measurement system (ZEM-3, ULVAC, Japan) in a helium atmosphere.

\section{RESULTS AND DISCUSSION}

The results of TEM investigation of the synthesized powders demonstrate a similar pattern for all compositions and show that the resulting material consists of irregularly shaped agglomerates with an average particle size of $\sim 20 \mathrm{~nm}$ (Fig. 1). However, annealing in argon at $260^{\circ} \mathrm{C}$ for $30 \mathrm{~min}$ leads to the formation of hexagonal particles with a diameter of $\sim 100 \mathrm{~nm}$ (see the inset in Fig. 1).

The X-ray phase analysis results are presented in Fig. 2. As follows from the figure, all as-prepared powders have the same rhombohedral crystalline structure of the $R-3 m$ space group and are characterized by the presence of elemental tellurium impurities (R3121 space group) with a concentration of $\sim 2$ at $\%$.

Compaction of the samples was carried out by the cold isostatic pressing method at a pressure of $250 \mathrm{MPa}$ with a holding time of $2 \mathrm{~min}$. The resulting compacts were annealed in an argon atmosphere at $410^{\circ} \mathrm{C}$ for 2 hours. The annealing temperature was selected taking into account the results of dilatometric investigations, which show that the sintering process terminates at a temperature of about $407^{\circ} \mathrm{C}$.

Annealing in argon does not affect the crystal-lattice parameters, but leads to the disappearance of elemental tellurium impurities (Fig. 2). X-ray phase analysis showed that all the materials are single-phase and have the hexagonal crystal structure $(R-3 m$ space group) after the annealing.

Calculations of the crystal-lattice parameters show that the introduction of doping atoms into the crystal

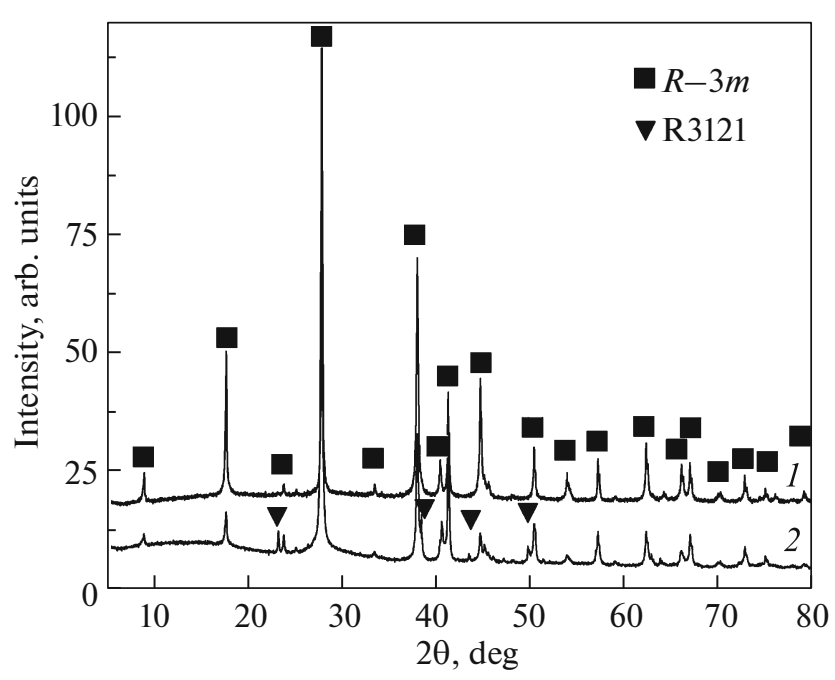

Fig. 2. Typical X-ray diffractograms for the as-prepared powders ( 1 ) and compacted annealed materials (2).

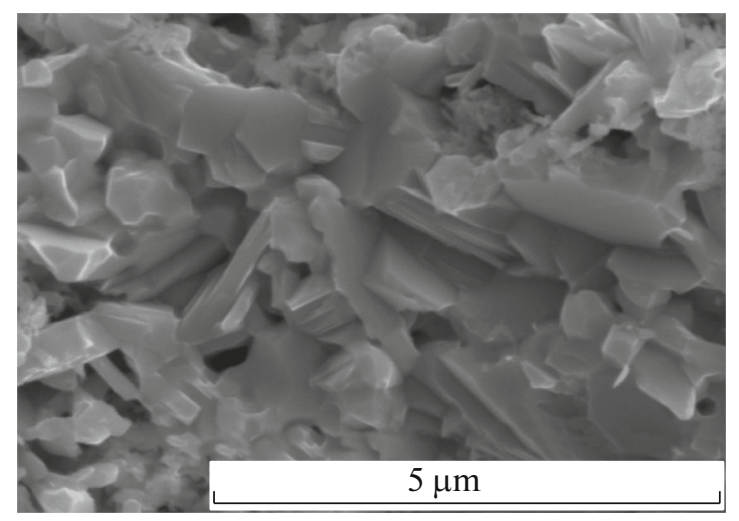

Fig. 3. Typical microstructure of the fracture planes of the compacted and annealed samples.

lattice of bismuth telluride does not lead to significant changes in these parameters, which could be associated with similar values of the covalent radius of bismuth and doping atoms.

SEM examination (Fig. 3) shows, that the samples have a layered structure with an average layer thickness of $80 \mathrm{~nm}$ and an average grain size of $1.6 \mu \mathrm{m}$. Pores with dimensions of $30 \mathrm{~nm}$ are observed along the grain boundaries, their formation at elevated temperatures may be associated, on the one hand, with the coagulation of point defects arising during the recrystallization process (vacancies), and, on the other hand, with a change in stoichiometry related to the evaporation of tellurium.

Figure 4 shows the temperature dependences of the electrical resistivity of the experimental samples. It was found that the doping of bismuth telluride with rare-earth elements can significantly reduce the electrical resistivity. At a temperature of $300 \mathrm{~K}$, the $\mathrm{Bi}_{2} \mathrm{Te}_{3}$ 


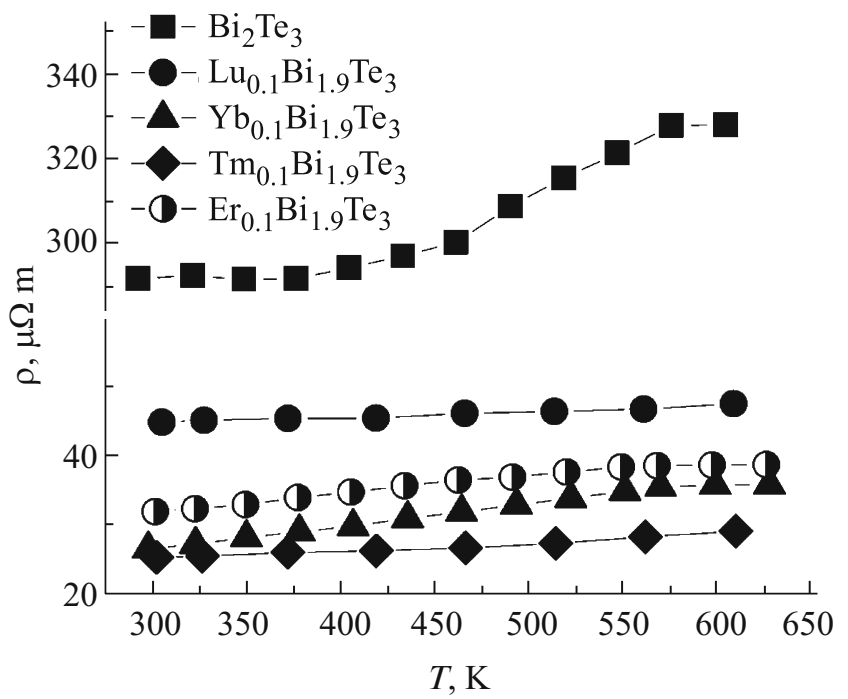

Fig. 4. Temperature dependences of the electrical resistivity of the investigated samples.

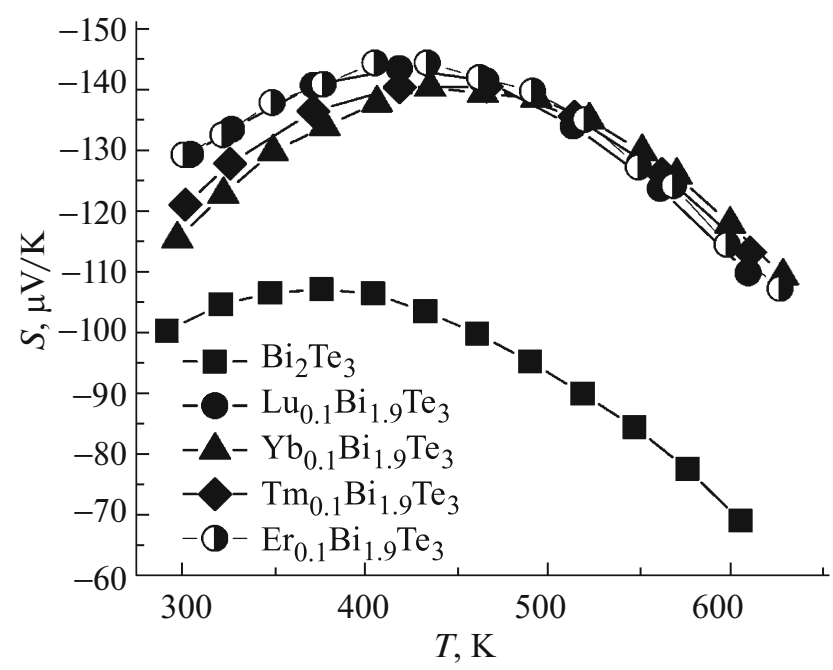

Fig. 5. Temperature dependences of the Seebeck coefficient of the investigated samples.

resistivity value is $290 \mu \Omega \mathrm{m}$, the resistivity values for $\mathrm{Lu}_{0.1} \mathrm{Bi}_{1.9} \mathrm{Te}_{3}, \mathrm{Yb}_{0.1} \mathrm{Bi}_{1.9} \mathrm{Te}_{3}, \mathrm{Tm}_{0.1} \mathrm{Bi}_{1.9} \mathrm{Te}_{3}, \mathrm{Er}_{0.1} \mathrm{Bi}_{1.9} \mathrm{Te}_{3}$ are of $45,27,25$ and $35 \mu \Omega \mathrm{m}$, respectively. Thus, the introduction of lanthanide atoms into the crystal lattice makes it possible to reduce the electrical resistivity by almost an order of magnitude.

Figure 5 shows the temperature dependences of the Seebeck coefficient $(S)$ of the investigated samples. It was found that all the curves have nonmonotonic behavior. As the temperature is raised, an increase in the Seebeck coefficient is observed; then, after reaching a maximum value, a gradual decrease is observed. Such nonmonotonic behavior of the temperature dependences for the Seebeck coefficient indicates the

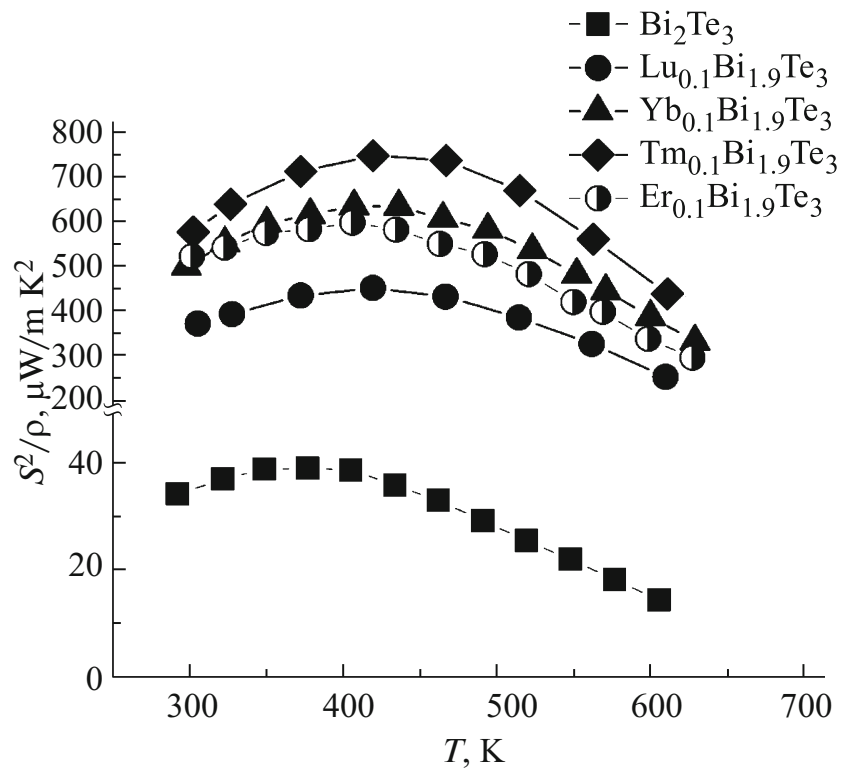

Fig. 6. Temperature dependences of the power factor of the investigated samples.

presence of two types of charge carriers ( $n$ - and $p$-type) in all the samples. However, taking into account the fact that the Seebeck coefficient in the entire temperature range for all the samples has a negative value, we can state that all the materials studied are $n$-type semiconductors.

It can be seen that there are no significant differences between the values of the Seebeck coefficient for the doped samples in the entire temperature range, but its value for the $\mathrm{Bi}_{2} \mathrm{Te}_{3}$ sample is much smaller. Hence, it can be concluded that the material with the smallest value of the electrical resistivity will have the largest value of the power factor among the doped samples, which is confirmed by the results shown in Fig. 6.

\section{CONCLUSIONS}

The $\mathrm{Bi}_{2} \mathrm{Te}_{3}$ and $\mathrm{R}_{0.1} \mathrm{Bi}_{1.9} \mathrm{Te}_{3}$ (where $\mathrm{R}=\mathrm{Er}, \mathrm{Tm}, \mathrm{Yb}$, $\mathrm{Lu}$ ) powders obtained by the microwave solvothermal synthesis method have a similar morphology and are represented by agglomerates of particles with an average size of $\sim 20 \mathrm{~nm}$. Additional annealing of the powder in argon at a temperature of $250^{\circ} \mathrm{C}$ leads to the formation of particles with hexagonal morphology and an average size of $\sim 100 \mathrm{~nm}$.

All the as-prepared powders have the same rhombohedral crystal structure of the $R-3 m$ space group and are characterized by the presence of elemental tellurium impurities ( 2 at $\%)$.

Compaction by cold isostatic pressing followed by annealing in an inert argon atmosphere allows nanostructured materials to be obtained. 
Annealing in argon does not affect the crystal-lattice parameters, but leads to the disappearance of elemental tellurium impurities.

It is shown that the doping of bismuth telluride with lanthanides has a positive effect on the functional electrophysical properties of the investigated compounds. It is established that the $\mathrm{Tm}_{0.1} \mathrm{Bi}_{1.9} \mathrm{Te}_{3}$ sample features the maximum value of the power factor. It can be assumed that an increase in the thermoelectric figure of merit of materials based on bismuth telluride doped with various lanthanides is possible with their simultaneous nanostructuring.

\section{REFERENCES}

1. L. P. Bulat, A. I. Drabkin, V. V. Karataev, V. B. Osvenskii, and D. A. Pshenai-Severin, Phys. Solid State 52, 1836 (2010).
2. A. A. Snarskii, A. K. Sarychev, I. V. Bezsudnov, and A. N. Lagarkov, Semiconductors 46, 659 (2012).

3. L. P. Bulat and D. A. Pshenai-Severin, Phys. Solid State 52, 485 (2010).

4. F. Wu, H. Z. Song, F. Gao, W. Y. Shi, J. F. Jia, and X. Hu, J. Electron. Mater. 42, 1140 (2013).

5. A. V. Shevelkov, Russ. Chem. Rev. 77, 1 (2008).

6. X. H. Ji, X. B. Zhao, Y. H. Zhang, B. H. Lua, and H. L. Ni, J. Alloys Compd. 387, 282 (2005).

7. F. Wu, H. Z. Song, J. F. Jia, F. Gao, Y. J. Zhang, and X. Hu, Phys. Status Solidi A 210, 1183 (2013).

8. W. Y. Shi, F. Wu, K. L. Wang, J. J. Yang, H. Z. Song, and X. Hu, J. Electron. Mater. 43, 3162 (2014).

9. J. Yang, F. Wu, Z. Zhu, L. Yao, H. Song, and X. Hu, J. Alloys Compd. 619, 401 (2015).

Translated by A. Melnikov 